QUIPURAMAYOC $\mid$ Revista de la Facultad de Ciencias Contables

Vol. 20 N. ${ }^{\circ} 37$ pp. 99-105 (2012) UNMSM, Lima, Perú

ISSN: 1560-9103 (versión impresa) / ISSN: 1609-8196 (versión electrónica)

\title{
EL PAPEL DEL ESTADO FRENTE A LA VIOLENCIA EN LOS CONFLICTOS SOCIALES EN PERÚ
}

\author{
THE ROLE OF THE STATE ADDRESS VIOLENCE IN \\ SOCIAL CONFLICTS IN PERU \\ JERI GLORIa Ramón RUFFNER* \\ Docente Principal de la Facultad de Ciencias Contables - UNMSM \\ [Recepción: Julio de 2012/ Conformidad: Agosto de 2012]
}

\section{RESUMEN}

La actual coyuntura social que se vive en el Perú actualmente pone en evidencia los distintos problemas recurrentes que por décadas no han sido resueltos y que se ha puesto en manifiesto a través de los llamados Conflictos Sociales. Algunas causales podrían resumirse como las necesidades básicas insatisfechas, la limitada participación en la vida pública de los grupos más vulnerables, la falta de mecanismos para plantear políticas de estado que permita una adecuada distribución de recursos; así como la falta de mecanismo para plantear demandas, entre otros, ante lo cual se necesita de nuevas y creativas formas de aproximarnos a los problemas para trabajar posibles soluciones que sean sostenibles en el tiempo.

Una de las condiciones básicas para la gobernabilidad del País es la implementación de políticas efectivas que estén orientadas al logro de la equidad y la inclusión, la misma que contribuirá a la promoción de inversiones y al proceso de descentralización. En este contexto, la adecuada y eficaz intervención del Estado en la gestión de los conflictos sociales resulta fundamental.

Es así que el presente artículo tiene por objetivo analizar las dimensiones del conflicto social; así como resaltar el papel del Estado y su competencia legal ante tales conflictos.

Palabras claves: Conflicto social, políticas integrales, demandas sociales, coyuntura social.

\begin{abstract}
The current social situation that exists today in Peru reveal various recurring problems that for decades have not been resolved and have resulted on the so-called Social Conflicts. Some of the causes can be summarized as unsatisfied basic needs, limited participation in the public life of the most vulnerable groups, lack of mechanism to raise estate policy that allows appropriate distribution of resources and a lack of mechanism to make demands. There is a need for new and creative ways to approach these problems. We need working solutions that are sustainable over time.

One of the basic conditions for the country's governability is the implementation of effective policies that are aimed at achieving equity and inclusion, which will also contribute to the promotion of investment and decentralization. In this context, appropriate and effective intervention in the management of social conflicts is essential. Thus, this article aims to analyze the dimensions of social conflict, and highlight the role of the state and its legal competence to such conflicts.
\end{abstract}

Keywords: Social conflict, comprehensive policies, social demand, social situation

* Doctora en Ciencias Contables y Magister en Contabilidad.Actual Directora Administrativa de la Facultad de Ciencias Contables-UNMSM. E-mail: jeriramon_162@hotmail.com 


\section{INTRODUCCIÓN}

Frente a la gran cantidad de conflictos sociales que se desarrollan a nivel regional y nacional, a menudo se reflexiona sobre el rol que deben cumplir los gobiernos regionales y locales frente a los conflictos sociales. Sin embargo, a pesar de ser conscientes de esta tarea en los últimos cinco años la Defensoría del Pueblo ha registrado el aumento del $300 \%$ en la frecuencia de estos conflictos.

Haciendo una investigación de las causales de los últimos conflictos sociales se puede saber que estos están fuertemente influenciados por la dinámica del crecimiento económico que ha tenido el país en los últimos años, pues esto ha implicado la analogía, necesariamente, que al haberse superado las expectativas económicas con ello debería establecerse medidas que generen el bienestar social y la representatividad política de ciertos sectores.

En efecto, según el reporte del Banco Central de Reserva (BCR), nuestro país ha tenido un crecimiento continuo durante los últimos diez años, con una tasa de promedio de PBI 7.15\% anual y de 20 años de modelo económico de mercado libre, y a pesar de que la pobreza ha disminuido ostensiblemente, hay todavía un sector de la población que vive en situación de pobreza y pobreza extrema, que no percibe que su calidad de vida haya mejorado substancialmente.

El cambio y la constante actividad d las inversiones también a despertado una demanda mayor en la protección de recursos como el agua y tierra, recursos que las comunidades consideran medios de subsistencia y que ahora ven amenazada su actividad económica. En la gran parte de la conflictividad social, se ve el tema de las empresas extractivas y el de la inversión extranjera, pero también existen otras causales de conflicto que guardan relación con los derechos laborales, los derechos políticos, la exigencia del adecuado uso de los recursos públicos que definitivamente conduce a que la población considere ausente y hasta a veces arbitrario a cierto sector del Estado.

Dentro de este contexto, el proceso de reforma de descentralización y de representación política juega un papel decisivo para el control de los problemas sociales; así mismo, la importancia de un modelo de gestión de los conflictos que permitan la confianza de la población en la administración publica que genere una cultura de dialogo y consenso.

\section{CARACTERÍSTICAS GENERALES DE LOS CONFLICTOS SOCIALES EN EL PERÚ}

Situaciones muy particulares que confronta la ciudadanía:

- La no construcción de una carretera,

- El temor a la contaminación del agua, la tierra o el aire,

- Las irregularidades en el manejo de los fondos públicos de parte de autoridades municipales,

- La falta de límites oficiales entre dos jurisdicciones, etc.

- Las desigualdades subsistentes, exclusiones que limitan o niegan el acceso a bienes y servicios, a las decisiones, a la valoración de las identidades particulares y que se expresan en las cifras de pobreza extrema rural,

- El acceso a una educación y salud de calidad.

- La fragmentación social,

- El racismo, la débil representación política, entre otros aspectos. 
Los conflictos por el nivel de manifiesto pueden ser: muy violentos, de larga duración y de una gran cohesión social. Estos son los elementos que preceden a movimientos políticos de mayor aliento, especialmente en contextos de identidades culturales fuertes y cada vez más conscientes de su exclusión. Un ejemplo de esta situación es perceptible en los movimientos sociales indígenas de la Amazonía peruana y de ciertas zonas de la Sierra sur.

A lo largo de los últimos cinco años, la Defensoría del Pueblo registró el aumento de más del $300 \%$ en la frecuencia de conflictos sociales. Dentro de este campo de conflictividad, la mayor cantidad corresponde a conflictos por temática socio ambiental. Así, por ejemplo, mientras que en el 2004 alcanzaban a 4 regiones del país, en el 2005 pasan a ser 7; el 2006, 12; durante el 2007, 15; en el 2008 alcanzaron a 19 regiones; en el 2009 a 23 y en el 2010 aumentó a 24 regiones. Pero, además, este incremento no solo corresponde al número de regiones, sino también a la intensidad.

\section{Grafico $\mathbf{N}^{\circ} 1$ \\ Distribución porcentual de conflictos sociales registrados}

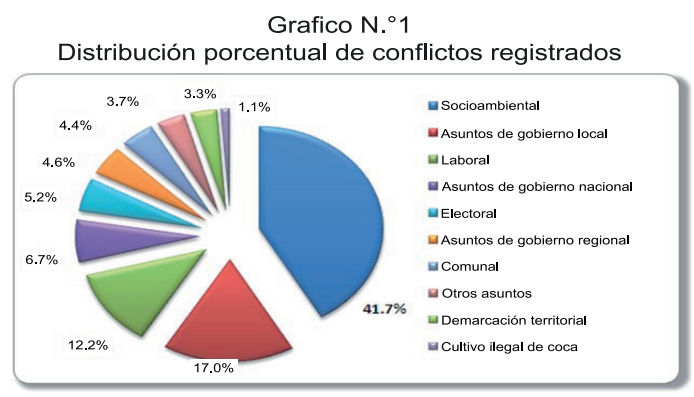

Fuente: Defensoría del Pueblo

Una de las características de los conflictos Sociales, a su vez es su forma de dar conocer los problemas y de llevar al espacio público determinadas demandas. El mecanismo de reclamación que se usa pasa en tiempos cortos a la protesta y la presión pública. A pesar de la intención dialogante de un sector del grupo social y su dirigencia, las exigencias de respuestas prontas y las limitaciones del Estado para proponer modalidades de diálogo creíbles y efectivas, precipitan el protagonismo de los actores violentos. Aunque el porcentaje de procesos de diálogo ha crecido en los últimos años de $32 \%$ en abril del 2008 a $53 \%$ en septiembre del 2011, de acuerdo al reporte No 91 de la Defensoría del Pueblo, el $58 \%$ de los procesos de diálogo se instala después de la violencia, porcentaje que con pequeñas variaciones se ha mantenido por lo menos desde enero del 2009.

Además, subsiste la idea de líderes organizacionales, dirigentes y organizaciones sociales la idea de que la violencia es un medio eficaz para alcanzar los objetivos. Permitiría acortar el desequilibrio que una de las partes percibe en su relación con la otra, es de utilización inmediata, transmite el mensaje del grupo con contundencia; infunde temor en las otras partes, da la impresión de cohesión e identidad en el grupo.

\section{FASE DE LOS CONFLICTOS SOCIA- LES:}

En el momento del Conflicto se puede identificar fases con características propias: temprana, escalamiento, crisis, desescalamiento y diálogo.

La secuencia de estas fases no se cumple necesariamente en el orden que aparece en el siguiente grafico pero guían el análisis de los casos en general: 


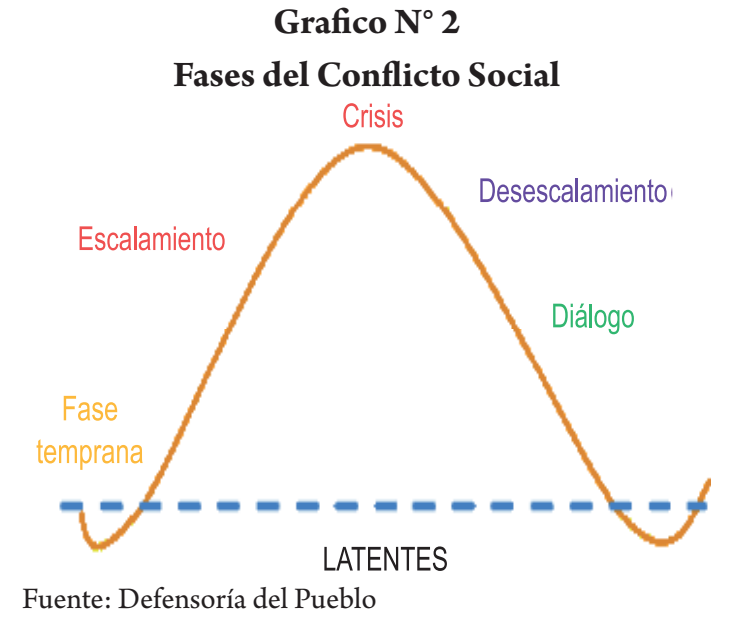

TIPOLOGÍA DE LOS CONFLICTOS SOCIALES:

Los conflictos sociales difieren unos de otros, ya sea por el contexto en que se desenvuelven, por los actores sociales, en relación a los demandas; sin embargo es posible clasificarlos a partir d determinados criterios.

En efecto, pueden ser agrupados según sus causas, las competencias de las instituciones llamadas a solucionarlos, por el ámbito territorial, por el impacto social, por su temática, por el acceso y distribución de recursos naturales, por temas de gobernabilidad, etcétera. También es posible clasificarlos por sus demandas, por las necesidades de los demandados, por fases del conflicto, por el estado del conflicto, etc.

\section{TIPOS:}

- Asuntos de Gobierno Local: La dinámica gira en torno a la gestión pública de los municipios provinciales y distritales.

- Asuntos de Gobierno Regional: La dinámica gira en torno a la gestión pública de los gobiernos regionales

- Asuntos de Gobierno Nacional: La dinámica gira en torno a la gestión pública del gobierno central.
- Socioambiental: La dinámica gira en torno al control, uso y/o acceso al ambiente y sus recursos. Están presentes también componentes políticos, económicos, sociales y culturales.

- Por demarcación territorial: La dinámica gira en torno al establecimiento de límites entre circunscripciones territoriales.

- Por cultivo ilegal de hoja de coca: La dinámica gira en torno a la siembra ilegal de coca, la presencia del narcotráfico y las acciones del Estado.

- Laborales: La dinámica gira en torno a los derechos laborales.

- Comunales: La dinámica gira en torno al acceso a recursos naturales, propiedad y límites territoriales entre comunidades.

- Electorales: La dinámica gira en torno a la no aceptación de resultados electorales y el rechazo a las autoridades electas.

- Otros conflictos: La dinámica gira en torno a reclamos estudiantiles, universitarios, etc.

\section{ALGUNAS CONSECUENCIAS DE LA VIOLENCIA EN LOS CONFLICTOS SOCIALES}

El efecto de los Conflictos sociales recaen en su mayoría en hechos de violencia y de las causas de los conflictos recaen sobre los derechos humanos, la gobernabilidad, el orden público, la seguridad y el establecimiento de una cultura democrática basada en el ejercicio del diálogo.

Es así que a través de un análisis de los conflictos sociales se puede evaluar el impacto de la violencia en los conflictos sociales, donde se desprende que durante la etapa de crisis de los conflictos, tanto civiles como políticas se convierten en sujetos agredibles. 


\section{Consecuencias en derechos humanos}

La violencia en conflictos sociales genera escenarios de riesgo en los que pueden producirse muertos y heridos. Las personas afectadas en sus derechos pueden ser tanto civiles como miembros de las fuerzas del orden.

Cabe llamar la atención en este punto sobre la idea de "costo social" como parámetro de medición de las intervenciones policiales. Si bien en las normas de la PNP, fuerzas del orden, no se da una definición operativa, el concepto se emplea para denotar que los planes de intervención se elaboran considerando el impacto negativo -sobre todo en las personas- que puede tener. En ese sentido, la vida humana por ser el bien más preciado que el Estado protege, no puede ser objeto de una evaluación cuantitativa, no es un "costo".

Un ejemplo de la afectación de los derechos fundamentales que se verifica en las situaciones de violencia en conflictos sociales se produce en torno a los terceros que no participan de los enfrentamientos. Muchas veces, sufren consecuencias sumamente graves, no solo en sus derechos colectivos a la paz y a la tranquilidad, sino también en su esfera más elemental, al violentarse sus derechos a la vida y a la salud, como consecuencia de las acciones de violencia (balaceras, pedreas, bloqueos de carreteras, principalmente) o las acciones de reposición del orden interno. Por ejemplo, en los conflictos sociales suscitados en torno a la creación de la Universidad de Tayacaja, en Huancavelica, en junio del 2011, donde fallecieron dos personas -incluso una menor de edad-, que no participaban en las acciones de protesta.

Por último, cabe anotar que -en un Estado cuyo fin supremo es la defensa de la persona humana y el respeto de su dignidad- estas afectaciones graves a los derechos humanos que se producen en tales escenarios generan un marco de responsabilidades, pues el Esta- do tiene la obligación de sancionar los actos que la sociedad considera más reprochables.

\section{Consecuencias sociales y políticas}

La lucha por una causa que el grupo social considera justa, produce una fuerte cohesión social y una memoria de los hechos que tiene efectos en el futuro. Si bien la identificación colectiva puede resultar positiva en términos de unidad de objetivos, no se puede ignorar que los actos de violencia pueden reforzar un tipo de aprendizaje social y político ajeno al ejercicio de una ciudadanía democrática.

En este sentido, la violencia en conflictos sociales tiene un impacto en la vida social y la cultura política: se afirman las conductas violentas; la imagen de la autoridad puede verse mellada en su capacidad de resolución de problemas y de protección de derechos; y se confunde el uso de las libertades que la Constitución Política garantiza con la realización de actos ilegales.

\section{Consecuencias económicas}

Las consecuencias económicas de la violencia en conflictos sociales presentan dimensiones micro y macro. En el aspecto micro se puede ubicar los impactos menos visibles: el cierre de pequeñas tiendas o puestos de venta debido al paro prolongado; la destrucción de pequeñas instalaciones (puestos de expendio de alimentos en vías públicas) que procuran el sustento económico familiar; la pérdida de oportunidades económicas; etc. Así también, el impacto en la economía familiar que producen las muertes o las lesiones, a veces permanentes, de algún miembro de la familia.

En términos macro, las consecuencias de la violencia en conflictos sociales se traducen en pérdidas a causa de la suspensión (momentánea o definitiva) de actividades mineras o hidrocarburíferas; de ingresos ge- 
nerados por el turismo; de horas/persona dejadas de laborar; del monto de los tributos no percibidos; pero, del mismo modo, de las transacciones no realizadas o de las oportunidades que se diluyen por la desconfianza que la violencia genera.

\section{EL PAPEL DEL ESTADO:}

El papel del estado es fundamental frente a las demandas sociales que surgen del conflicto social, en la medida en la que esta tenga implicancia en materia de derechos fundamentales ya sea en relación a la educación, salud, protección del medio ambiente, etc.

Para tal tarea el Estado debe asumir diferentes tipos de obligaciones. Esto es, no restringir de manera arbitraria el ejercicio de los derechos humanos, adoptar medidas que garanticen la seguridad de terceros ante conflictos, así como implementar medidas progresivas que permitan su realización efectiva.

Cuando el conflicto social deriva en hechos de violencia, la afectación al bienestar se acentúa, en tanto se podrían vulnerar otros derechos fundamentales, como la integridad, el libre tránsito, la propiedad, la seguridad e incluso la vida.

El Estado debe de "garantizar la plena vigencia de los derechos humanos", consagrado en la Constitución Política, conforme al cual la defensa de la persona humana y el respeto de su dignidad constituyen el fin supremo de la sociedad y del Estado.

En este marco constitucional, el Estado está llamado a preservar a la persona humana -de forma individual y a la población como colectivo-, libre de amenazas o afectaciones a su vida, a su salud y, en general, a su integridad física y psíquica, para lo cual debe mantener incólume la esfera y el equilibrio social del ser humano, principalmente por medio de medidas Preventivas.

\section{CONCLUSIONES:}

1.- Los conflictos sociales se comportan como modalidades de expresión ciudadana que con una adecuada gestión, pueden convertirse en oportunidades de cambio para el país.

2.- El país experimenta un incremento de conflictos sociales que pone ene evidencia elementos de debilidad en la intermediación política, en la capacidad de gestión publica y en las practicas de dialogo.

3.- El estado, es sus tres niveles de gobierno y sus instituciones autónomas ha ido incorporando progresivamente el tema de la conflictividad social como una de sus prioridades; en embargo, la capacidad de gestionar, de prevenir y transformar conflictos no es aún optima ante la envergadura de los problemas.

4.- Los procedimientos de dialogo son complementarios del ejercicio regular de los procedimientos legales, cuya eficacia depende de las reformas integrales en políticas publicas que el Estado proponga, estas políticas necesariamente deben ser de gran alcance.

5.- El mayor número de hechos de violencia se reporta en los conflictos socioambientales.

6.- La violencia propia de conflictos sociales trae consecuencias negativas en la vida social y política del país. La violencia reiterada refuerza comportamientos agresivos y la idea equivocada que es un medio eficaz para obtener respuesta, todo lo contrario, esta desgasta el logro de objetivos, desgasta la intermediación política, debilita la autoridad democrática y el sistema de reglas de derecho. Asimismo, confunde el uso delas libertades que la constitución garantiza con la realización de actos ilegales. 
7.- El estado tiene responsabilidad frente al conflicto social y las posibles situaciones de violencia. Cada problema que da origen al conflicto debe generar en el Estado un papel activo y una competencia legal por resolverlo.

\section{REFERENCIAS BIBLIOGRÁFICAS:}

1.- Burton, John W. (2000) La resolución de conflictos como sistema político. Instituto de Análisis y Resolución de Conflictos, George Mason University, Fairfax, Virginia.

2.- Ertel, Danny (1996) Negociación 2000; La Colleccion de Conflict Management Santa Fe de Bogota. McGraw Hill.
3.- Fisher, Roger, William Ury and Bruce Patton (1995) Si... de Acuerdo! Bogotá: Grupo Editorial Norma.

4.- Folberg, Jay y Alison Taylor (1992) Mediación: Resolución de conflictos sin litigo México Limusa.

5.- Folger, Joseph P. y Tricia S.Jones (1997) Nuevas direcciones en mediación Barcelona, España. Paidos

6.- Galtung, Johan (1998) Tras de Violencia: Reconstrucción, Reconciliación y Resolución España BAKEAZ.

7.- Informe de la Defensoría del Pueblo Conflictos Sociales - Resolución Defensorial N. ${ }^{\circ} 009-2012 / \mathrm{DP}$ 